\title{
Comparison is beyond IPASS and OPTIMAL
}

\author{
${ }^{1}$ KC Lam *, FHKAM (Medicine), ${ }^{2}$ Tony SK Mok, FHKCP, FHKAM (Medicine) \\ ${ }^{1}$ Department of Clinical Oncology, Prince of Wales Hospital, Shatin, Hong Kong \\ ${ }^{2}$ Department of Clinical Oncology, The Chinese University of Hong Kong, Shatin, Hong Kong \\ *kc_lam@clo.cuhk.edu.hk
}

Hong Kong Med J 2014;20:176-7

DOI: $10.12809 / \mathrm{hkmj} 144267$

Now that first-line epidermal growth factor receptor tyrosine kinase inhibitor (EGFR TKI) is recognised as a standard therapy for non-small-cell lung cancer (NSCLC) with activating mutations, ${ }^{1-7}$ it is only natural for some of us to ask which TKI is the best. In the absence of a direct head-to-head randomised controlled study, Lee et $\mathrm{al}^{8}$ used an indirect comparison method to compare the efficacy and cost-effectiveness of gefitinib and erlotinib, results of which are published in this issue. The authors concluded that erlotinib has better efficacy and is more cost-effective than gefitinib. ${ }^{8}$ Is this a trustworthy conclusion?

For reason that is not entirely clear to us, authors chose to use data from only the OPTIMAL and IPASS studies. Truth is that there are four randomised studies on gefitinib (IPASS, FIRST-SIGNAL, NEJ002, and WJTOG 345) and three randomised studies on erlotinib (OPTIMAL, EURTAC, and ENSURE) in patients with activating EGFR mutation-positive NSCLC. The authors excluded EURTAC study from the comparison because of the differences in baseline demographic data and ethnicity. Similar to IPASS, EURTAC is a registration study using stringent criteria for documentation of treatment outcomes and toxicity. The OPTIMAL study was not considered a registration study by the China Food and Drug Administration, and that was exactly the reason for the replication of OPTIMAL study (ENSURE study) in China. Grade 3 or above skin rash rate was higher in the EURTAC study (13\%) as compared with IPASS study (3.1\%), and this is a fact that should not be ignored. The median progressionfree survival (PFS) and hazard ratio were comparable between EURTAC and IPASS and, again, this fact was ignored. Lee et $\mathrm{al}^{8}$ should incorporate data from more relevant clinical trials into the indirect comparison to provide a non-biased estimate of the true treatment effect. As erlotinib is slightly more expensive than gefitinib in the Hong Kong public health care system, it will be unrealistic to conclude a better cost-effectiveness if the two drugs were shown to have similar efficacy in an honest manner.

Kim et $\mathrm{al}^{9}$ conducted a prospective openlabel randomised non-comparative parallel study in a single Korean hospital to evaluate the efficacy of erlotinib and gefitinib in those EGFR mutationpositive NSCLC patients or patients with at least two out of three clinical factors associated with higher incidence of EGFR mutations who failed platinumbased chemotherapy. In this exploratory comparison, there were no statistically significant differences in response rate and median PFS between the two drugs. More treatment-related grade- 3 or -4 adverse events were observed with erlotinib versus gefitinib (12.4\% vs $4.2 \%)$. Wu et al ${ }^{10}$ conducted a retrospective study to evaluate the difference in efficacy between erlotinib and gefitinib in Taiwanese patients with advanced-stage EGFR mutation-positive NSCLC. They also found no statistically significant difference in response rate and PFS between the two drugs. Lim et $\mathrm{al}^{11}$ performed a retrospective review to examine the treatment outcomes with two EGFR TKIs with a match-pair case-control study design. Again, there were no statistically significant differences in response rates, PFS, and overall survival between the two drugs. An ongoing prospective randomised trial in China compares erlotinib with gefitinib in NSCLC patients harbouring EGFR exon 21 mutation. This will be the only true head-to-head comparison between gefitinib and erlotinib in this setting.

While we argue about which is a better EGFR TKI, treatment paradigm is shifting to second and third generations of EGFR TKIs. Afatinib, an irreversible pan-HER inhibitor, has been proven to be superior to standard platinum-based chemotherapy. ${ }^{12}$ LUX-Lung 7 trial, a randomised phase IIb study comparing afatinib to gefitinib in EGFR mutation-positive NSCLC, is completed and results are pending. Furthermore, ARCHER 1050 will be the first randomised phase III study comparing dacomitinib with gefitinib in patients with either exon 19 or 21 mutations. Phase I studies on thirdgeneration EGFR TKIs including AZ9291 and CO1686 have also reported high tumour response rate in patients with resistant T790M mutation. Its role as first-line EGFR TKI remains to be explored.

Treatment of advanced-stage lung cancer is rapidly evolving. Instead of asking the question of which is a better EGFR TKI, perhaps we should focus on how to improve outcomes for our future patients.

\section{References}

1. Mok TS, Wu YL, Thongprasert S, et al. Gefitinib or carboplatin-paclitaxel in pulmonary adenocarcinoma. $\mathrm{N}$ Engl J Med 2009;361:947-57. 
2. Maemondo M, Inoue A, Kobayashi K, et al. Gefitinib or chemotherapy for non-small-cell lung cancer with mutated EGFR. N Engl J Med 2010;362:2380-8.

3. Mitsudomi T, Morita S, Yatabe Y, et al. Gefitinib versus cisplatin plus docetaxel in patients with non-small-cell lung cancer harbouring mutations of the epidermal growth factor receptor (WJTOG345): an open label, randomised phase 3 trial. Lancet Oncol 2010;11:121-8.

4. Zhou C, Wu YL, Chen G, et al. Erlotinib versus chemotherapy as first-line treatment for patients with advanced EGFR mutation-positive non-small-cell lung cancer (OPTIMAL, CTONG-0802): a multicentre, open-label, randomised, phase 3 study. Lancet Oncol 2011;12:735-42.

5. Rosell R, Carcereny E, Gervais R, et al. Erlotinib versus standard chemotherapy as first-line treatment for European patients with advanced EGFR mutation-positive nonsmall-cell lung cancer (EURTAC): a multicentre, openlabel, randomised phase 3 trial. Lancet Oncol 2012;13:23946.

6. Han JY, Park K, Kim SW, et al. First-SIGNAL: first-line single-agent iressa versus gemcitabine and cisplatin trial in never-smokers with adenocarcinoma of the lung. J Clin Oncol 2012;30:1122-8.

7. Wu YL, Zhou C, Wu G, et al. Quality of Life (QoL) analysis from ENSURE, a phase 3, open-label study of first-line erlotinib versus gemcitabine/cisplatin (gp) in Asian patients with EGFR mutation-positive NSCLC. J Thorac Oncol 2014;9:7S-52S.

8. Lee VW, Schwander B, Lee VH. Effectiveness and costeffectiveness of erlotinib versus gefitinib in first-line treatment of epidermal growth factor receptor-activating mutation-positive non-small-cell lung cancer patients in Hong Kong. Hong Kong Med J 2014;20:178-86.

9. Kim ST, Uhm JE, Lee J, et al. Randomized phase II study of gefitinib versus erlotinib in patients with advanced nonsmall cell lung cancer who failed pervious chemotherapy. Lung Cancer 2012;75:82-8.

10. Wu WS, Chen YM, Tsai CM, et al. Erlotinib has better efficacy than gefitinib in adenocarcinoma patients without EGFR-activating mutations, but similar efficacy in patients with EGFR-activating mutations. Exp Ther Med 2012;3:207-13.

11. Lim SH, Lee JY, Sun JM, et al. Comparison of clinical outcome between gefitinib and erlotinib treatment in patients with non-small cell lung cancer harbouring an epidermal growth factor receptor exon 19 or exon 21 mutations [abstract]. J Clin Oncol 2013;31 Suppl:e19051.

12. Sequist LV, Yang JC, Yamamoto N, et al. Phase III study of afatinib or cisplatin plus pemetrexed in patients with metastatic lung adenocarcinoma with EGFR mutations. J Clin Oncol 2013;31:3327-34. 